\title{
Diagnostic yield and predictive factors of findings in small-bowel capsule endoscopy in the setting of iron-deficiency anemia
}

\section{다 (1) $(2)$}

\author{
Authors \\ Carolina Olano, Ximena Pazos, Karla Avendaño, Alfonso Calleri, Carlos Ketzoian
}

Institution

Clinica de Gastroenterología “Prof. Henry Cohen”, Hospital de Clínicas, UDELAR, Montevideo, Uruguay

submitted 27.9.2017

accepted after revision 20.2.2018

\author{
Bibliography \\ DOI https://doi.org/10.1055/a-0593-5915 | \\ Endoscopy International Open 2018; 06: E688-E693 \\ (c) Georg Thieme Verlag KG Stuttgart · New York \\ ISSN 2364-3722
}

Corresponding author

Carolina Olano, MD MSc, Hospital de Clinicas, Av. Italia s/n

CP 11600, Montevideo, Uruguay

Fax: +598-2-4872572

carolinaolanouruguay@gmail.com

\section{ABSTRACT}

Introduction Despite the widespread use of small-bowel capsule endoscopy (CE), there is still limited data on its utility and effectiveness for the diagnosis and management of patients with iron-deficiency anemia (IDA).

Aim To assess the diagnostic yield of CE and the factors predicting positive findings in patients with IDA.
Methods Patients with unexplained IDA and negative upper and lower endoscopy were included. A positive diagnostic yield was considered when CE diagnosed one or more lesions that could explain the IDA. Sex, age, NSAID consumption, blood transfusion requirement, and ferritin and hemoglobin levels were recorded.

Results In total, 120 CE were included (mean age 58.5 years; F/M 82:38). Mean hemoglobin levels were $9 \mathrm{~g} / \mathrm{dL}$ and mean ferritin levels were $15.7 \mathrm{ng} / \mathrm{mL}$. Positive findings were present in $50 \%$ of patients. The most frequent was angiodysplasia (45\%). Despite several baseline variables being significantly associated with positive findings, using a logistic regression model, it was verified that male sex (OR 3.93; $95 \% \mathrm{Cl} 1.57-9.86)$, age (OR $1.03 ; 95 \% \mathrm{Cl} 1.00-1.06)$, and hemoglobin levels (OR $0.73 ; 95 \% \mathrm{Cl} 0.57-0.94$ ) were the variables having an independent effect on the probability of obtaining positive findings. Age older than 50 years (OR $14.05 ; 95 \% \mathrm{Cl} 1.69-116.23$ ) and male sex (OR 3.63; $95 \% \mathrm{Cl}$ $1.29-10.17$ ) were the variables which increased the risk of diagnosing angiodysplasia.

Conclusions CE is a useful technique in patients with IDA. To improve its yield, it is necessary to select patients carefully. Male sex, older age, and low hemoglobin levels were associated with a risk of positive finding in this group of patients. The risk of diagnosing angiodysplasia increased with male sex and older age.

\section{Introduction}

Iron-deficiency anemia (IDA) occurs in $5 \%$ of premenopausal women and $1-2 \%$ of men and postmenopausal women. It is a common reason for referral to gastroenterologists, accounting for $4-13 \%$ of outpatient visits [1,2]. IDA may be due to: blood loss (gastrointestinal, hemoptysis, traumatic, menorrhagia, hematuria, regular donors, post-surgery, iatrogenic repeated studies, etc.), malabsorption (celiac disease, gastrectomy or other enteropathies), or low iron intake (very low frequency in adults in our area) [1,2]. In premenopausal women, the most common cause is gastrointestinal and/or gyneco-obstetric blood loss and in men and postmenopausal women, the most common cause is gastrointestinal blood loss. In most cases, the cause of bleeding is accessible to standard endoscopy (gastroscopy and colonoscopy) [1,2]. However, in 2-10\%, the lesions are located in the small intestine, so that these studies are negative [1,2].

Small-bowel capsule endoscopy (CE) is a diagnostic tool that allows painless and safe assessment of the small bowel, leading to an etiologic diagnosis of small-bowel bleeding in approximately $60 \%$ of cases $[3,4]$. Two meta-analyses [4,5] have shown that CE is superior to push enteroscopy and radiological imaging for diagnosing clinically significant small-bowel pathology in patients with "suspected small-bowel bleeding (SBB)", previously called obscure gastrointestinal bleeding 
(OGIB). Furthermore, CE and double-balloon enteroscopy have comparable diagnostic yields in SBB $[6,7]$.

In an attempt to assess the relevance of findings of CE, Saurin et al. classified the lesions as highly relevant (P2), of uncertain relevance $(\mathrm{P} 1)$, or of low relevance (P0) [8]. P2 lesions include angiodysplasia, tumors, ulcerative lesions, and varices, P1 include red spots and small or isolated erosions, and P0 include visible mucosal veins, diverticula without the presence of blood, or nodules without mucosal breaks. In a subsequent study, a P3 category was included for active bleeding [9].

Several studies have evaluated the effectiveness of $C E$ in SBB, but few have focused on IDA alone [10 - 17]. Furthermore, in those few reports, the exact significance of the lesions identified has been poorly studied [14,15]. Therefore, we aimed to assess the diagnostic yield of CE in patients with IDA who had undergone previous thorough work-up. We also aimed to determine whether sex, age, NSAIDs, blood transfusions, hemoglobin $(\mathrm{Hb})$, or ferritin levels could be predictors of findings.

\section{Patients and methods}

This is a retrospective cohort study of all consecutive patients undergoing CE for the evaluation of unexplained IDA conducted at the Gastroenterology Department of the "Hospital de Clínicas", a tertiary referral center in Uruguay. All patients with unexplained IDA who had undergone at least one negative upper and lower endoscopy between January 2008 and May 2016 were included. IDA was defined, according to the laboratory indices, as hemoglobin $(\mathrm{Hb})$ less than $13 \mathrm{~g} / \mathrm{dL}$ in men and $12 \mathrm{~g} / \mathrm{dL}$ in women, along with a serum ferritin level less than $50 \mathrm{ng} / \mathrm{mL}$. Negative endoscopy was considered when no lesions that explained IDA were found or when found, they did not explain the severity of IDA.

Exclusion criteria included patients aged below 18 years, Crohn's disease, pregnancy, with gynecological causes for IDA or celiac disease. Gynecological causes were excluded by clinical history and gynecological exam. Celiac disease was defined as the presence of at least one positive antibody (EmA or TTG) and/or any grade of villous atrophy and increased intraepithelial lymphocytes in duodenal biopsies.

The following variables were collected in a standardized data sheet: age, sex, NSAID consumption, blood transfusion requirement, hemoglobin, ferritin, and results of upper and lower endoscopy. A positive diagnostic yield was considered to be one or more lesions that were believed to be a definitive cause of IDA (P2 or P3 of the Saurin classification).

\section{Small-bowel capsule endoscopy procedure}

Informed consent was obtained from all participants, and CE was carried out using the Capsule Endoscopy System (Medtronic, United States). PillCamSB, SB2, and SB3 capsules were used, and videos were analyzed using Rapid Reader, version 5 , 7, and 8, respectively (Medtronic, United States). As none of the patients included were suspected of having a small-bowel stricture or known or suspected Crohn's disease, a patency capsule (dissolvable radio-opaque capsule) was not performed before CE. On the afternoon before the study, all patients had only liquids and some patients (mostly the first studies performed) also received a prep solution (Precolsur, Celsius, Uruguay) containing sodium sulfate, sodium bicarbonate, sodium chloride, and potassium chloride. All patients fasted the night before the procedure. A sensor array was worn externally and the capsule was swallowed. Patients were allowed to drink clear fluids 2 hours after swallowing the capsule and to eat a light lunch 4 hours after. The capsule passed with a normal bowel movement and was discarded. The sensor recorded images for 8 hours with SB type capsules, and until the capsule reached the colon (real time viewer) with SB2 or SB3 type capsules. The images were uploaded as a video and interpreted by a single experienced endoscopist (CO).

\section{Statistical analysis}

A descriptive analysis was performed, with the presentation of absolute frequencies and relative measures. Our unit of analysis was the endoscopic study, and the same patient may have needed more than one endoscopic study at different times. For quantitative variables, measures of central tendency and dispersion were used. The chi-squared test was used to contrast between nominal and/or ordinal variables.

Significance tests were used to compare continuous variables, and previous verifications of the conditions of application of the tests. Normality tests were performed to study the distribution of these variables. The significance level in all cases was 0.05 . When the variables did not follow a normal distribution, non-parametric tests were used to study their association with angiodysplasia and the presence of findings (Mann-Whitney $U$ test). The analysis of age was made as a continuous variable and also in two groups (older and younger than 50 years). This cut-off was selected following Muhammad et al. [13]. A multivariate analysis of the variables whose $P$ value $\leq 0.25$ was performed. A logistic regression was performed using as dependent variables: presence of findings and the presence of angiodysplasia.

\section{Results}

In total, 120 CE were performed in 118 patients. CE completion rate was $96.7 \%$, defined by visualization of the cecum on the recording. Four studies (3.3\%) were defined as incomplete, in one of them CE was stopped after 3 hours due to an ulcerated stenosis that was later crossed through; in another patient CE stopped at a submucosal lesion and stayed there for 5 hours but was defecated 24 hours later. In the remaining two cases, the sensor was removed 8 hours after being swallowed (misreading of cecal image at data recorder screen). There were no complications. Of the total, $51 \mathrm{CE}$ were performed with PillCamSB, 51 CE with PillCamSB2, and 18 with PillCamSB3.

The mean patient age was 58.5 years, ranging from 18 to 86 years. Eighty-seven patients $(72.5 \%)$ were older than 50 years. Eighty-two were women (68.3\%). Fifty-four patients (45\%) used NSAIDs and 54 patients (45\%) had received blood transfusions ( $\triangleright$ Table 1$)$. Only 10 patients $(8.3 \%$ ) were receiving anticoagulant therapy. The mean $\mathrm{Hb}$ level was $9 \mathrm{~g} / \mathrm{dL}$ (range $4-$ $11.9 \mathrm{~g} / \mathrm{dL}$ ). Twenty-one patients $(17.5 \%)$ had severe anemia 
- Table 1 Characteristics of the total population with IDA and those with and without positive findings.

\begin{tabular}{|c|c|c|c|c|}
\hline & Total n (\%) & $\begin{array}{l}\text { Positive findings } \\
(n=60)\end{array}$ & $\begin{array}{l}\text { Without positive findings } \\
(n=60)\end{array}$ & $P$ value \\
\hline Sex: male & $38(32 \%)$ & $27(45 \%)$ & $11(18 \%)$ & 0.02 \\
\hline Age $>50$ years & $87(72.5 \%)$ & $50(83 \%)$ & $37(62 \%)$ & NS \\
\hline \multicolumn{5}{|l|}{ Anemia, g/dL } \\
\hline - Mild-moderate $(\mathrm{Hb}>7)$ & $99(83 \%)$ & $45(75 \%)$ & $54(90 \%)$ & NS \\
\hline - Severe $(\mathrm{Hb} \leq 7)$ & $21(17 \%)$ & $15(25 \%)$ & $6(10 \%)$ & NS \\
\hline \multicolumn{5}{|l|}{ Ferritin, ng/dL } \\
\hline . $<15$ & $68(57 \%)$ & $35(58 \%)$ & $33(55 \%)$ & NS \\
\hline - $15-30$ & $37(31 \%)$ & $18(30 \%)$ & $19(32 \%)$ & NS \\
\hline - $31-50$ & $15(12 \%)$ & $7(12 \%)$ & $8(13 \%)$ & NS \\
\hline NSAID: yes & $54(45 \%)$ & $30(50 \%)$ & $24(40 \%)$ & NS \\
\hline Transfusion: yes & $54(45 \%)$ & $33(55 \%)$ & $21(35 \%)$ & 0.03 \\
\hline Anticoagulant therapy: yes & $10(8 \%)$ & $4(7 \%)$ & $6(10 \%)$ & NS \\
\hline Capsule reached cecum & $116(97 \%)$ & $57(95 \%)$ & $59(98 \%)$ & NS \\
\hline
\end{tabular}

$(\mathrm{Hb} \leq 7 \mathrm{~g} / \mathrm{dL})$ and 99 patients $(82.5 \%)$ had mild to moderate anemia. The mean ferritin level was $15.7 \mathrm{ng} / \mathrm{dL}$ (range $1.5-$ $48.0 \mathrm{ng} / \mathrm{dL})$.

Positive findings were present in 60 patients with IDA(50\%). The distribution of positive findings is shown in Fig. 1 and images of their spectrum are shown in $\mathbf{F i g . 4}$. Two patients (3.3\%) had more than one lesion. Active small-bowel bleeding was found in 5 patients ( $8.3 \%), 3$ of them without identification of a clear lesion. Three patients (5\%) had positive findings outside the small bowel (colonic active bleeding, pyloric ulcer, and cecal angiodysplasia) and were included as positive findings for statistical analysis.

In the 3 patients with bleeding lesions outside the small bowel, the following interventions were performed: a second colonoscopy was performed in the patient with colonic active bleeding and a previously missed colon cancer was diagnosed; upper gastrointestinal endoscopy was performed to take gastric and pyloric ulcer biopsies and a colonoscopy was performed to treat cecal angiodysplasias with argon plasma coagulation (APC).

Demographic, clinical, and laboratory data of patients with and without positive findings are shown in $>$ Table 1.

Male sex $(P=0.02)$ and transfusion requirement $(P=0.028)$ were statistically significantly associated with positive findings.

In a multivariable analysis using a logistic regression model, it was verified that male sex (OR 3.93; $95 \% \mathrm{Cl} 1.57-9.86)$, age (OR 1.03; $95 \% \mathrm{Cl} 1.00-1.06$ ), and hemoglobin (OR 0.73; $95 \% \mathrm{Cl}$ $0.57-0.94$ ) were the variables having an independent effect on the probability of obtaining positive findings in patients with IDA ( $\triangleright$ Fig. 2 and $\triangleright$ Fig. 3 ). Age older than 50 years (OR 14.05; $95 \% \mathrm{Cl} 1.69-116.23$ ) and male sex (OR 3.63; $95 \% \mathrm{Cl} 1.29-$ 10.17 ) were the variables which increased the risk of diagnosing angiodysplasia.

\section{Discussion}

In this study, the diagnostic yield of CE for IDA was $50 \%$, which is in agreement with data from previous studies [17]. Our study was a phase IV clinical study, confirming the high diagnostic yield of CE found in previous phase II clinical trials $[18,19]$. Most studies on CE had been performed in the USA, Europe, and the Far East. Our study is the largest on CE in IDA in South America. Therefore, our data also help define the epidemiological and etiological characteristics of IDA in additional geographic areas.

The majority of positive findings in our study were due to angiodysplasia and non-specific inflammation/ulceration, which is consistent with those found in similar studies from USA and Europe but not those from Asia. In Asia, reports of small-bowel tumors, inflammatory lesions, and multifocal stenosis enteritis are more common [20-23]. It has been recognized that CE is more sensitive than other modes of small-bowel imaging for the detection of vascular malformations [24].

All lesions included were considered to be highly relevant lesions (P2 of the Saurin classification). The "Saurin classification of small-bowel lesions on CE" allows risk stratification of patients [8,9]. Highly relevant lesions (P2) had previously been validated by two blinded endoscopists in $100 \%$ of cases, compared with $73 \%$ and $27 \%$ of cases for intermediate- (P1) and low-relevance (P0) lesions, respectively [25]. There was a necessity for therapy in a significantly higher number of P2 lesions (61\%), compared with P1 or P0 lesions (23\%) [25].

In this series, in addition to angiodysplasia, one case of multiple phlebectasias was included as vascular lesions. These are rare benign vascular anomalies that may present with occult or overt small-bowel bleeding. We have already reported two 


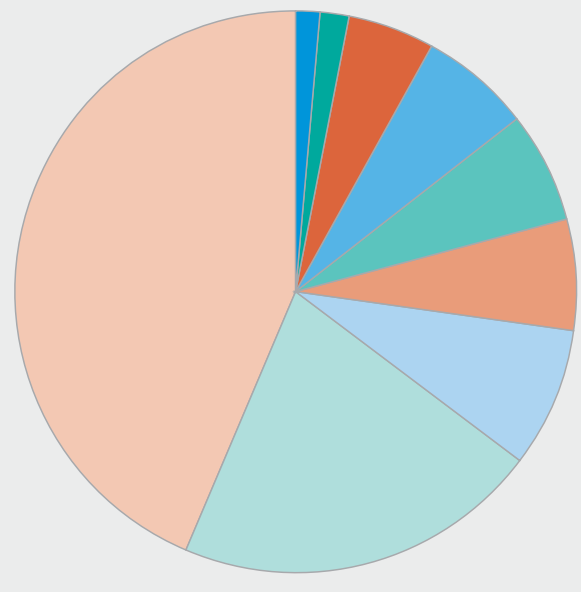

Diaphragmlike stricture; 1

Multiple phlebectasia; 1

Findings outside SB; 3

Ulcerated polyps; 4

Malignant tumor like lesions; 4

Ulcerated submucosal lesions; 4

Active bleeding; 5

Nonespecific inflammation and ulceration; 13

Angiodysplasia; 27

Fig. 1 Distribution of positive findings in 60 patients with IDA.

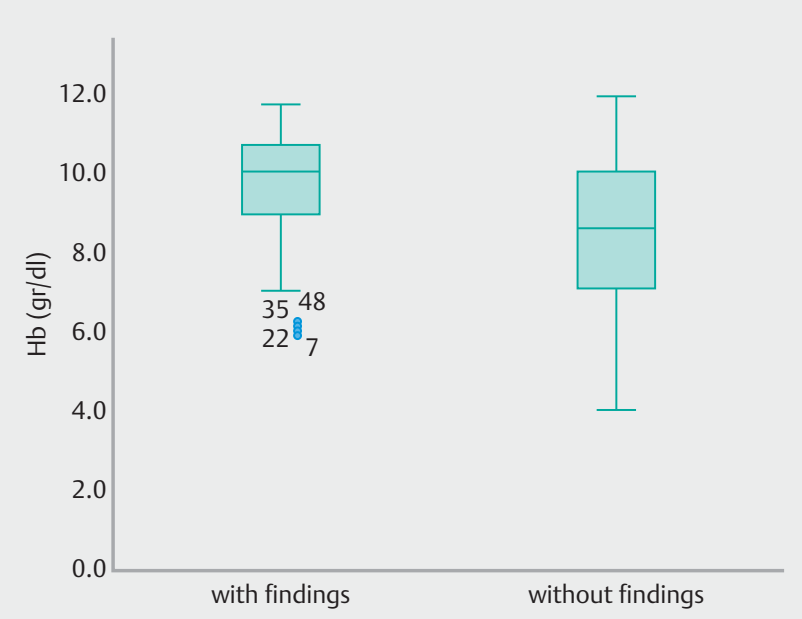

- Fig. 2 Box plots of Hb levels in patients with and without positive findings.

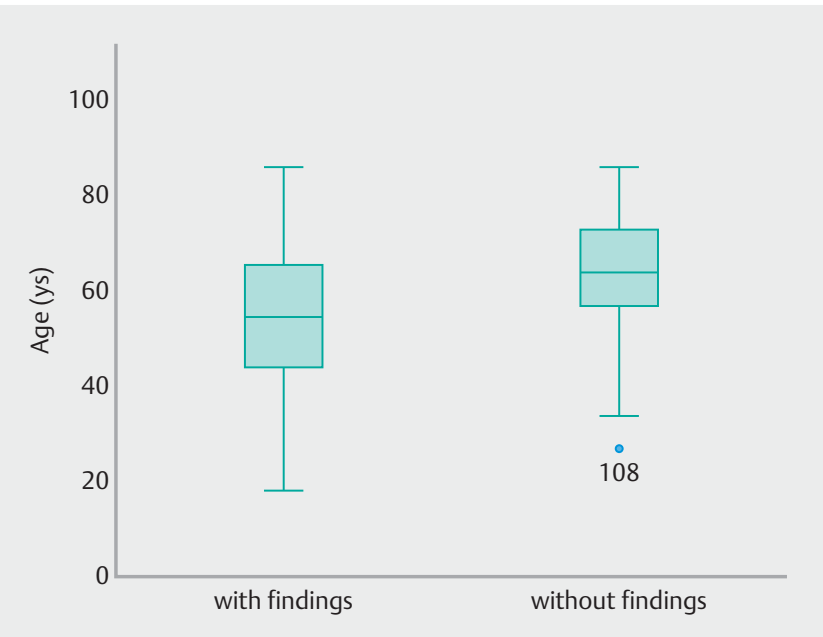

- Fig. 3 Box plots of age in patients with and without positive findings. cases of these lesions, even with active bleeding during the procedure [26].

Due to the clinical relevance, in the $50 \%$ diagnostic yield, we included $4.4 \%$ of findings outside the small bowel. Our lesion miss rate outside the small bowel is much lower than those reported in previous studies, which found that patients referred for small-bowel endoscopic imaging had a significant number of lesions within the reach of upper and lower endoscopy [2729]. Fry et al. [30] found $24.3 \%$ of sources of gastrointestinal bleeding outside the small bowel with double balloon enteroscopy (DBE). Elijah et al. [31] also found that up to $38.8 \%$ of patients undergoing CE for OGIB had significant lesions of the upper gastrointestinal tract such as gastric antral vascular ectasias (GAVE), gastric ulcers, and Cameron's lesions. These lesions were evidently missed during electrocardiography (EGC) and colonoscopy performed before DBE and/or CE. In our series, the lesions missed were: a pyloric ulcer whose histology revealed an adenocarcinoma, a colonic active bleeding due to a small colorectal cancer (CRC), and a cecal angiodysplasia. We believe that having performed the previous upper and lower endoscopies in our tertiary center may have resulted in a lower number of missed lesions. Indeed, despite the reported high percentages of lesions outside the small bowel and the high cost of the $\mathrm{CE}$, most guidelines still do not routinely support a second-look endoscopy prior to small-bowel CE [32 - 34].

There is limited published data in which clinical and laboratory factors predict the ability of CE to detect pathology in IDA. In a large series of patients with OGIB of which $74 \%$ had IDA, Siduh et al. [33] found that increasing age, use of warfarin, and liver comorbidity were significant factors which predicted a higher yield with CE. More recently, in 221 patients with IDA, Koulaouzidis et al. [17] found that age, prior blood transfusions, use of anticoagulants, and male sex were factors associated with a higher diagnostic yield for all types of small-bowel pathology. Similar to Koulaouzidis et al., we found that increas- 

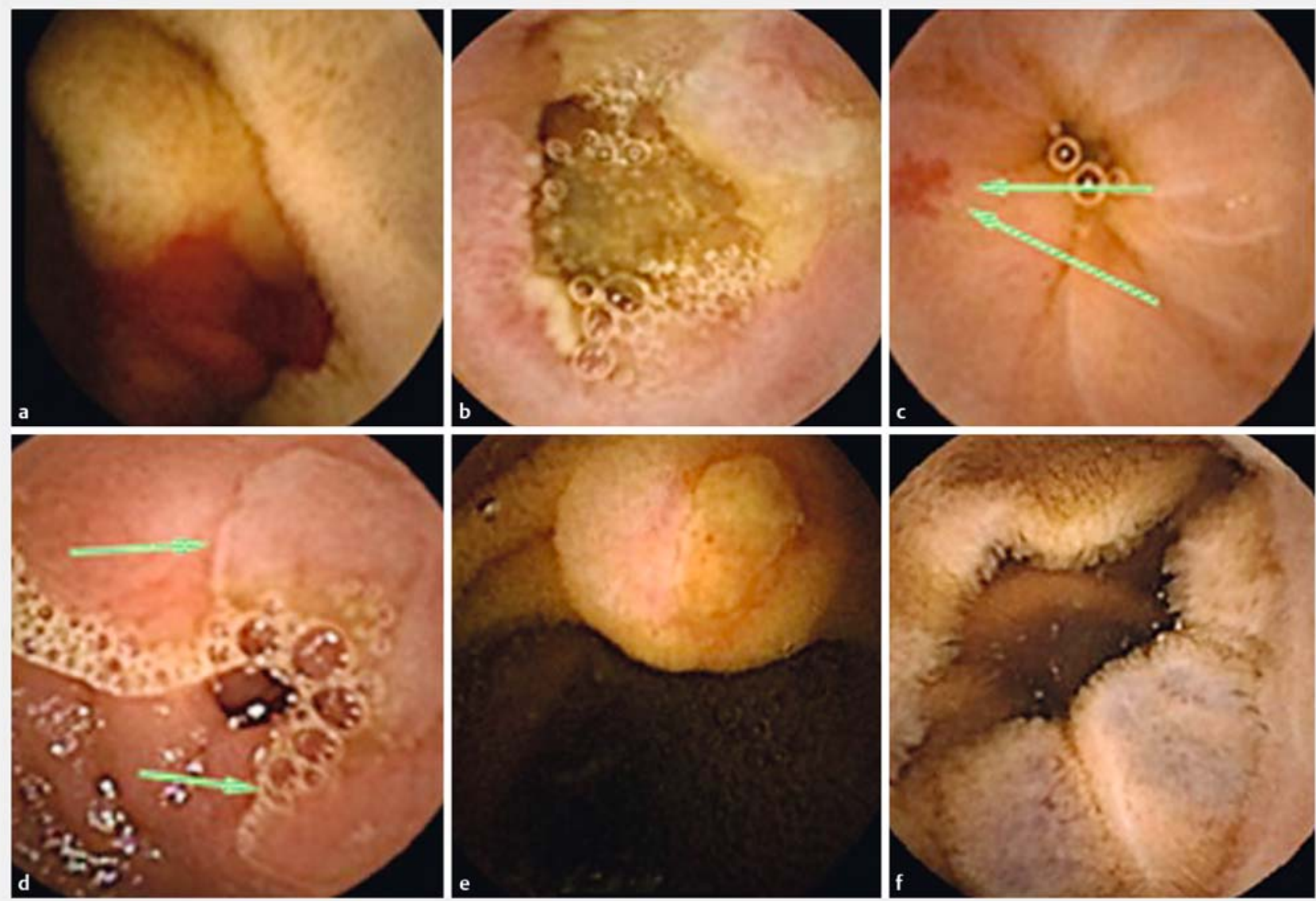

Fig. 4 Spectrum of positive findings. a Active bleeding; b malignant tumor-like lesion; c angiodysplasia; $\mathbf{d}$ ulcerated stenosis; e ulcerated submucosal lesion; $\mathbf{f}$ multiple phlebectasia.

ing age was associated with a higher detection rate of smallbowel angiodysplasia.

All these results are partially in line with ours. Nevertheless, and to the best of our knowledge, ours is the first study to show low hemoglobin levels as a predictor of findings in patients with IDA. Furthermore, in contrast to most previous studies, our study focused exclusively on patients with IDA. We carefully select the IDA group, excluding celiac disease diagnosis in all patients by antibody detection and/or duodenal biopsies. Thus, this study demonstrated that, in IDA patients, after an intensive previous work-up, capsule endoscopy would be a very useful technique to find lesions in elderly patients with severe anemia.

Our study has several potential limitations. First, it is a retrospective study and thus prone to the potential deficits of such design. However, we carefully collected all variables including all videos of the procedures to ensure a complete database. Second, we are a tertiary center and our findings may not be reproduced by others. Third, as most of the patients were referred exclusively for $\mathrm{CE}$, some data is missing. We only had data for $H$. pylori investigation in $29 \%$. As $H$. pylori infection can be a cause of unexplained iron-deficiency anemia, this could be a limitation. Also, data on iron supplementation was not available. Fourth, we included nonspecific diagnosis as potentially explaining the bleeding leading to IDA. But until now there is no clear definition of which lesions result in bleeding and we based our definitions of bleeding lesions on existing classifications and guidelines. Nonetheless, further studies will be mandatory to clearly define which lesions results in bleeding IDA. Finally, patients were referred only for CE from different healthcare centers and the subsequent medical treatment depended on the attending physician and the availability of resources in their health service. It was not possible to perform a complete follow-up of these patients so an analysis of outcomes was not included in the study. It would be interesting to perform a study on this variable in the future.

In conclusion, CE is a useful technique in patients with IDA. However, as shown by this study, to improve its yield, it is necessary to select patients carefully. Male sex, old age, and low hemoglobin levels increased the risk of positive findings in this group of patients. The risk of diagnosing angiodysplasia increases with age. Furthermore, large multicenter, phase IV studies are needed to better define the lesions causing IDA and the outcomes of patients with IDA subjected to CE. 
Competing interests

None

References

[1] Bermejo F, Garcia-Lopez S. A guide to diagnosis of iron deficiency and iron deficiency anemia in digestive diseases. World J Gastroenterol 2009; 15: $4638-4643$

[2] Goddard AF, Mclntyre AS, Scott BB. Guidelines for the management of iron deficiency anaemia. British Society of Gastroenterology. Gut 2000; 46: IV1 - IV5

[3] Teshima CW, Kuipers EJ, van Zanten SV et al. Double balloon enteroscopy and capsule endoscopy for obscure gastrointestinal bleeding: an updated meta-analysis. J Gastroenterol Hepatol 2011; 26: 796 801

[4] Leighton JA, Triester SL, Sharma VK. Capsule endoscopy: a meta-analysis for use with obscure bleeding and Crohn's disease. Gastrointest Endosc Clin N Am 2006; 16: 229-250

[5] Triester SL, Leighton JA, Leontiadis Gl et al. A meta-analysis of the yield of capsule endoscopy compared to other diagnostic modalities in patients with obscure gastrointestinal bleeding. Am J Gastroenterol 2005; 100: $2407-2418$

[6] Pérez-Cuadrado-Robles E, Esteban-Delgado P, Martínez-Andrés B et al. Diagnosis agreement between capsule endoscopy and doubleballoon enteroscopy in obscure gastrointestinal bleeding at a referral center. Rev Esp Enferm Dig 2015; 107: 495 - 500

[7] Chen X, Ran ZH, Tong JL. A meta-analysis of the yield of capsule endoscopy compared to double-balloon enteroscopy in patients with small bowel diseases. World J Gastroenterol 2007; 13: 4372-4378

[8] Saurin JC, Delvaux M, Gaudin JL et al. Diagnostic value of endoscopic capsule in patients with obscure digestive bleeding: blinded comparison with video push-enteroscopy. Endoscopy 2003; 35: 576- 584

[9] Rahmi G, Samaha E, Vahedi K et al. Long-term follow-up of patients undergoing capsule and double-balloon enteroscopy for identification and treatment of small-bowel vascular lesions: a prospective, multicenter study. Endoscopy 2014; 46: 591 - 597

[10] Yamada A, Watabe H, Yamaji Y et al. Incidence of small intestinal lesions in patients with iron deficiency anemia. Hepatogastroenterology $2011 ; 58: 1240-1243$

[11] Riccioni ME, Urgesi R, Spada C et al. Unexplained iron deficiency anaemia: is it worthwhile to perform capsule endoscopy? Dig Liver Dis 2010; 42: 560 - 566

[12] Apostolopoulos P, Liatsos C, Gralnek IM et al. The role of wireless capsule endoscopy in investigating unexplained iron deficiency anemia after negative endoscopic evaluation of the upper and lower gastrointestinal tract. Endoscopy 2006; 38: $1127-1132$

[13] Muhammad A, Pitchumoni CS. Evaluation of iron deficiency anemia in older adults: the role of wireless capsule endoscopy. J Clin Gastroenterol 2009; 43: 627-631

[14] Holleran GE, Barry SA, Thornton OJ et al. The use of small bowel capsule endoscopy in iron deficiency anemia: low impact on outcome in the medium term despite high diagnostic yield. Eur J Gastroenterol Hepatol 2013; 25: 327-332

[15] Koulaouzidis A, Yung DE, Lam J et al. The use of small bowel capsule endoscopy in iron deficiency anaemia alone; be aware the young anaemic patients. Scand J Gastroenterol 2012; 47: 1094-1100

[16] Tong J, Svarta S, Ou G et al. Diagnostic yield of capsule endoscopy in the setting of iron deficiency anemia without evidence of gastrointestinal bleeding. Can J Gastroenterol 2012; 26: 687-690
[17] Koulaouzidis A, Rondonotti E, Giannakou A et al. Diagnostic yield of small-bowel capsule endoscopy in patients with iron-deficiency anemia: a systematic review. Gastrointest Endosc 2012; 76: 983 - 992

[18] Delvaux M, Fassler I, Gay G. Clinical usefulness of the endoscopic videocapsule as the initial intestinal investigation in patients with obscure digestive bleeding: validation of a diagnostic strategy based on the patient outcome after 12 months. Endoscopy 2004; 36: 1067 1073

[19] Scarpa F, Jacob H, Lewkowicz S et al. Initial experience of wirelesscapsule endoscopy for evaluating occult gastrointestinal bleeding and suspected small bowel pathology. Am J Gastroenterol 2001; 97 : $2776-2779$

[20] He YF, Hao NB, Yang WC et al. Small bowel endoscopy diagnostic yield and reasons of obscure GI bleeding in Chinese patients. Gastroenterol Res Pract 2014; 2014: 437693

[21] Shinozak S, Yamamoto H, Yano T et al. Long-term outcome of patients with obscure gastrointestinal bleeding investigated by double-balIoon endoscopy. Clin Gastroenterol Hepatol 2010; 8: 151-158

[22] Matsumoto T, lida M, Matsui T et al. Chronic nonspecific multiple ulcers of the small intestine: a proposal of the entity from Japanese gastroenterologists to Western enteroscopists. Gastrointest Endosc 2007; 66: S99-S107

[23] Chang DK, Kim JJ, Choi H et al. Double balloon endoscopy in small intestinal Crohn's disease and other inflammatory diseases such as cryptogenic multifocal ulcerous stenosing enteritis (CMUSE). Gastrointest Endosc 2007; 66: S96-\$98

[24] Hakim FA, Alexander JA, Huprich JE et al. CT-enterography may identify small bowel tumors not detected by capsule endoscopy: eight years experience at Mayo Clinic Rochester. Dig Dis Sci 2011; 56: $2914-2919$

[25] Saurin JC, Delvaux M, Vahedi K et al. Clinical impact of capsule endoscopy compared to push enteroscopy: 1-year follow-up study. Endoscopy 2005; 37: 318-323

[26] Olano C, Machado P, Berrueta J et al. Obscure gastrointestinal bleeding due to small-bowel phlebectasias. Endoscopy 2014; 46: E223224

[27] Akin FE, Yurekli OT, Bolat AD et al. Analysis of non-small bowel lesions detected by capsule endoscopy in patients with potential small bowel bleeding. Diagn Ther Endosc 2016; 2016: 9063293

[28] Fry LC, Belluti M, Neumann $\mathrm{H}$ et al. Incidence of bleeding lesions within reach of conventional upper and lower endoscopes in patients undergoing double-balloon enteroscopy for obscure gastrointestinal bleeding. Aliment Pharmacol Ther 2009; 29: $342-349$

[29] Elijah D, Daas A, Brady P. Capsule endoscopy for obscure GI bleeding yields a high incidence of significant treatable lesions within reach of standard upper endoscopy. J Clin Gastroenterol 2008; 42: 962-963

[30] Gerson LB, Fidler JL, Cave DR et al. ACG Clinical Guideline: Diagnosis and management of small bowel bleeding. Am J Gastroenterol 2015; 110: $1265-1287$

[31] Enns RA, Hookey L, Armstrong D et al. Clinical practice guidelines for the use of video capsule endoscopy. Gastroenterology 2017; 152: 497-514

[32] Pennazio M, Spada C, Eliakim R et al. Small-bowel capsule endoscopy and device-assisted enteroscopy for diagnosis and treatment of small-bowel disorders: European Society of Gastrointestinal Endoscopy (ESGE) Clinical Guideline. Endoscopy 2015; 47: 352 - 376

[33] Sidhu R, Sanders DS, Kapur K et al. Factors predicting the diagnostic yield and intervention in obscure gastrointestinal bleeding investigated using capsule endoscopy. J Gastrointest Liver Dis 2009; 18: 273 278

[34] Muhammad A, Vidyarthi G, Brady P. Role of small bowel capsule endoscopy in the diagnosis and management of iron deficiency anemia in elderly: A comprehensive review of the current literature. World J Gastroenterol 2014; 20: 8416-8423 